\title{
BMPs functionally replace KIf4 and support efficient reprogramming of mouse fibroblasts by Oct4 alone
}

\author{
Jiekai Chen ${ }^{1, *}$, Jing Liu ${ }^{1,}$, Jiaqi Yang ${ }^{1}$, You Chen ${ }^{1}$, Jing Chen ${ }^{1}, \mathrm{Su} \mathrm{Ni}^{1}$, Hong Song ${ }^{1}$, Lingwen Zeng ${ }^{1}$, Ke Ding ${ }^{1}$, \\ Duanqing Pei $^{1}$ \\ ${ }^{I}$ Key Laboratory of Regenerative Biology, South China Institute for Stem Cell Biology and Regenerative Medicine at Guangzhou \\ Institutes of Biomedicine and Health, Chinese Academy of Sciences, Guangzhou 510530, China
}

Generation of induced pluripotent stem cells by defined factors has become a useful model to investigate the mechanism of reprogramming and cell fate determination. However, the precise mechanism of factor-based reprogramming remains unclear. Here, we show that Klf4 mainly acts at the initial phase of reprogramming to initiate mesenchymal-to-epithelial transition and can be functionally replaced by bone morphogenetic proteins (BMPs). BMPs boosted the efficiency of Oct4/Sox2-mediated reprogramming of mouse embryonic fibroblasts (MEFs) to $\sim 1 \%$. BMPs also promoted single-factor Oct4-based reprogramming of MEFs and tail tibial fibroblasts. Our studies clarify the contribution of Klf4 in reprogramming and establish Oct4 as a singular setter of pluripotency in differentiated cells.

Keywords: Oct4; iPSCs; reprogramming; stem cell; BMPs; MET

Cell Research (2011) 21:205-212. doi:10.1038/cr.2010.172; published online 7 December 2010

\section{Introduction}

Somatic cells can be reprogrammed into induced pluripotent stem cells (iPSCs) by ectopic expression of Oct4, Sox2, Klf4 and c-Myc [1-6]. However, the Yamanaka factors included Klf4 and Myc both not directly implicated in pluripotency, but did not have the pluripotency regulator Nanog, suggesting that there is a mechanistic difference between induction and maintenance of pluripotency [1]. We recently reported that mouse fibroblasts undergo a mesenchymal-to-epithelial transition (MET) orchestrated by Oct4, Sox 2 and Myc to suppress snail expression and blunt TGF- $\beta$ signaling, and Klf4 to activate expression of epithelial markers, such as $C d h 1$, during the initial phase of reprogramming [7], thus, defining a concrete molecular and cellular step towards pluripotency $[7,8]$. The division of labor among the Yamanaka factors suggests that each factor plays distinct

\footnotetext{
*These two authors contributed equally to this work. Correspondence: Duanqing Pei

Tel: +86-20-3201-5201

E-mail: pei_duanqing@gibh.ac.cn

Received 20 October 2010; accepted 25 October 2010; published online 7 December 2010
}

roles during the reprogramming process. Taken together, one would expect that Oct4 alone can reprogram somatic cells such as mouse fibroblasts into pluripotency as Oct4 governs embryonic cell fate $[9,10]$. In this study, we analyzed the relative contribution of Sox 2 and Klf4 towards reprogramming under a chemically defined system optimized for robust reprogramming. We showed that Klf4 plays an important role in triggering MET, which can also be initiated by bone morphogenetic proteins (BMPs) $[8,11]$. Then, we demonstrated that Oct4/Sox2 (OS) and Oct4 alone can reprogram mouse embryonic fibroblasts (MEFs) and tail tibial fibroblasts (TTFs) efficiently in the presence of BMPs.

\section{Results}

Klf4 mainly acts at the initial phase of reprogramming

Multifactorial requirement is a great barrier for mechanistic investigation of factor-based reprogramming. However, the function of each factor employed in reprogramming is poorly understood and at least two factors are required (including ectopic and endogenous expression) in the literature [7, 12-16]. Among the four Yamanaka factors, c-Myc was proven dispensable and can be replaced by various methods [17-20]. Oct4, Sox2 
and Klf4 are currently considered as indispensable factors, as replacement of them would greatly reduce reprogramming efficiency. OK-mediated reprogramming of mouse fibroblasts was reported with moderate efficiency, by using compounds targeting various pathways such as Bayk8644, CHIR99021, RepSox and A83-01 [7, 12, 13, $16,21]$. By contrast, OS-mediated reprogramming was reported only with p53-null fibroblasts [14]. We previously reported that OKS-mediated reprogramming can be enhanced by the optimized serum-free medium iSF1 [19]. Further optimization led to improved efficiency of OKS-mediated reprogramming and we found that iPSCs can be generated by either OK or OS with very low efficiency (Supplementary information, Figure S1A) in a newly formulated medium iCD1. The resulting OS or OK iPSCs were similar to ESCs and could be differentiated into three germ layers in teratoma assay as expected (Supplementary information, Figure S1B). Remarkably, OK-mediated reprogramming appeared to be more efficient and more rapid than OS (Supplementary information, Figure S1A).

We then examined the requirement of Klf4 or Sox2 in a doxycycline-inducible system. We found that Klf4 is required mostly during the initial phase of reprogramming (Figure 1A). We performed transcriptomic analysis and showed that Klf4 upregulated genes important for epithelial development (Figure 1B). Consistent with this analysis, OK stimulates robust expression of epithelial markers (such as Cdh1 and Occludin), while OS has little effect on them (Figure 1C). As MET is an early requisite step during the reprogramming of MEFs and as Klf4 participates in MET by activating the epithelial program [7], we conclude that MET is a rate-limiting step in OSmediated reprogramming (Figure 1D).

BMPs functionally replace Klf4 and support efficient reprogramming with $O S$

BMPs have been implicated in MET by reversing TGF- $\beta$-induced EMT in NP1 cells and reported to enhance MET during reprogramming $[8,11]$, and thus may functionally substitute Klf4. To test this possibility, we showed that BMP4 and BMP7 can enhance the expression of epithelial genes $(C d h 1, E p C A M$, etc.) and inhibit the expression of mesenchymal genes (Zeb1, Twist1, etc.) in OS-infected MEFs, but cannot promote epithelial markers further in OK-infected cultures (Figure 2A and $2 \mathrm{~B}$ and Supplementary information, Figure S2A and S2B). These results indicate that BMPs can trigger MET in OS-induced reprogramming, but cannot further enhance the strong MET activated by Klf4 in OK reprogramming. Consistently, BMP4 greatly improved the kinetics and efficiency of OS reprogramming (up to $\sim 0.8 \%$ at day 15, a 400-fold plus 9 days improvement), but had no effect on OK reprogramming and even had inhibitory effect on OKS reprogramming (Figure 2C and 2D and Supplementary information, Figure S2C). We also showed that BMP2, BMP6, BMP7 and BMP9, similar to BMP4, significantly enhanced the OS-mediated reprogramming (Figure 2E, and Supplementary information, Figure S2D). We also examined the treatment windows of BMP4 in OS-mediated reprogramming and showed that exposure of BMP4 at days 3-9 is most effective (Figure 2F).

We then investigated the role of Cdh1 in OS-mediated reprogramming and showed that knockdown of Cdh1 decreased reprogramming efficiency significantly (Supplementary information, Figure S2E), suggesting that the observed enhancement by BMP4 is Cdh1 dependent. We also tested other compounds including Kenpaullone [22] and showed that none of them can enhance OSmediated reprogramming (Supplementary information, Figure S2F). As expected, OS-iPSC clones derived with BMP4 expressed pluripotent markers such as Oct4, Nanog, Rex1 and SSEA-1 (Figure 2G). We then showed that these OS iPSCs contributed to the generation of chimeric mice, which underwent germline transmission (Figure $2 \mathrm{H}$ ), indicating that the OS iPSCs were fully reprogrammed.

\section{BMPs induce efficient Oct4-mediated reprogramming by promoting $M E T$}

We then hypothesized that BMPs should enhance Oct4-mediated reprogramming by inducing MET. As in OS-mediated reprogramming, BMP4 and BMP7 improved the expression of Cdh1 and EpCAM in Oct4transduced MEFs (Figure 3A and Supplementary information, Figure S3A). We then showed that Oct4 alone was able to convert MEFs into iPSCs efficiently in $\mathrm{iCD} 1+\mathrm{BMP} 4$ with reasonable kinetics $(\sim 0.05 \%$ at D24 post-infection; Figure 3B). Under the same culture condition, we showed that adult mouse TTFs can also be reprogrammed by Oct 4 alone reproducibly, demonstrating that Oct4 is sufficient to induce adult somatic cells to pluripotency (Figure 3C-3E). We also showed that BMPs has no effect on the expression level of p53 and p21 (Supplementary information, Figure S3B) and further showed that no other tested chemical compounds can support Oct4-mediated reprogramming (Supplementary information, Figure S3C).

We then characterized the Oct4-iPSC clones isolated with iCD1+BMP4 and showed that they expressed pluripotent markers Oct4, Nanog, Rex1, SSEA-1, Dppa3 and Dnmt31 (Figure 4A and 4B, and Supplementary information, Figure S3D). All Oct4-iPSC clones that we obtained 
A
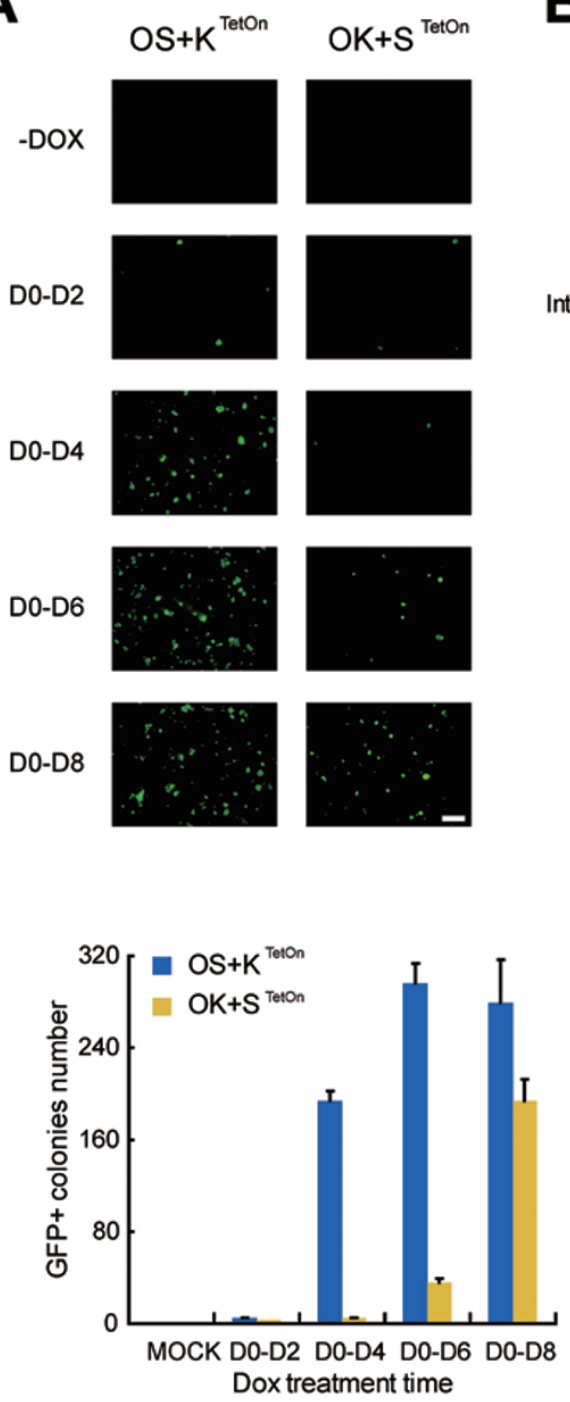

B

Gene ontology analysis of Klf4 downstream genes

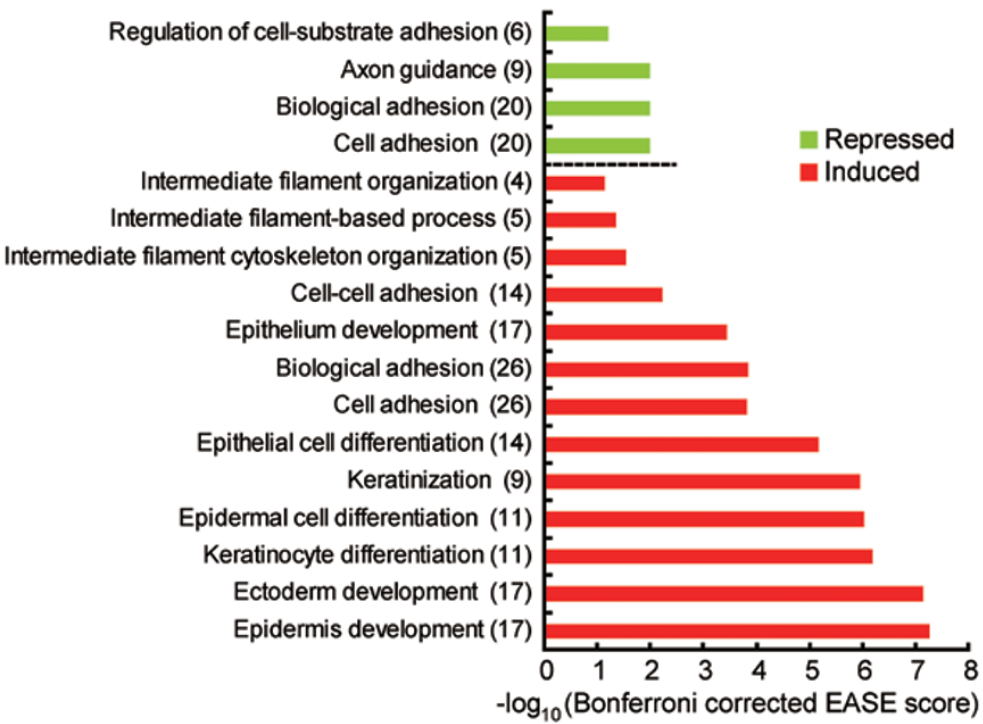

C

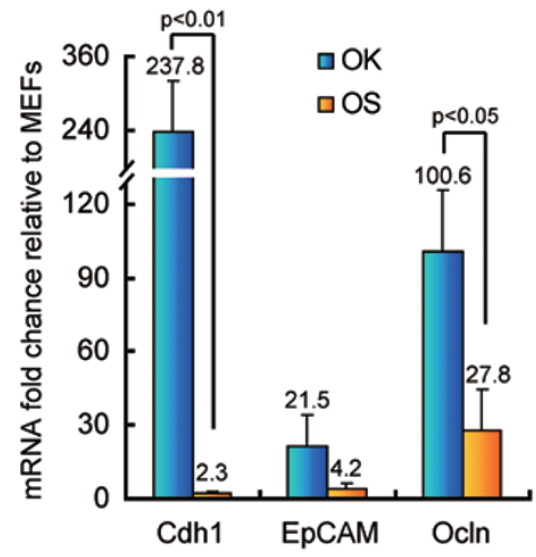

D
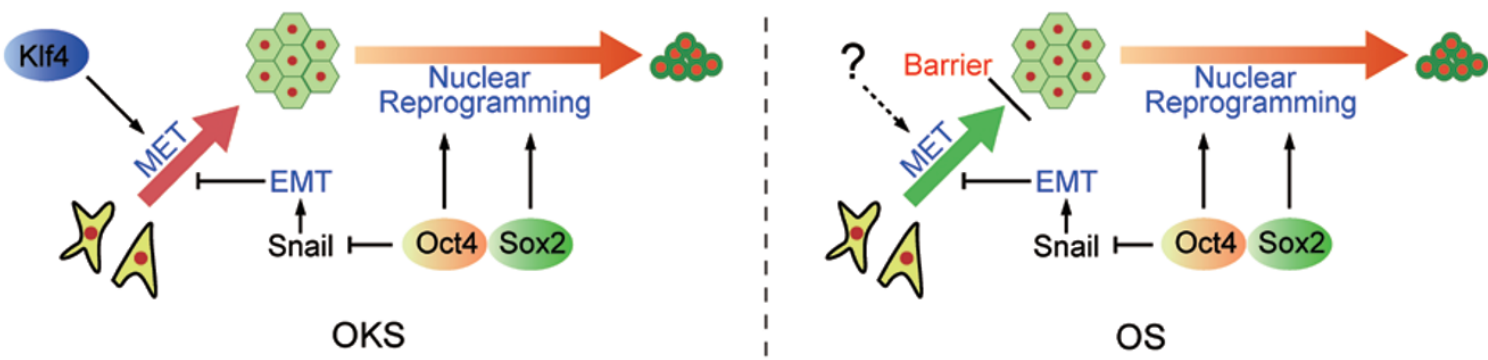

Figure 1 KIf4 mainly acts at early MET phase of reprogramming. (A) Klf4 is critical in the initial stage of reprogramming. By using inducible system, ectopic expression of KIf4 or Sox2 in OKS-mediated reprogramming was terminated in different days. Representative photos were taken and reprogramming efficiency was scored at day 12. $n=3$. Scale bar, $2 \mathrm{~mm}$. (B) Klf4 mainly regulated cell-cell adhesion and induced epithelial markers. MEFs transduced by retroviral OS and lentiviral TetOnKIf4 were cultured in iCD1 with or without doxycycline for 3 days and analyzed by DNA microarray. Gene ontology analysis (Bonferroni corrected EASE scores were presented) based on the DE list (expression change > three folds), number in brackets indicated the number of genes including in DE list. (C) Expression of epithelial marks in OK- or OS-infected MEFs at post-infection day 3. $n=3$. (D) Hypothesis of KIf4 and Sox2's function in the course of reprogramming. 
A

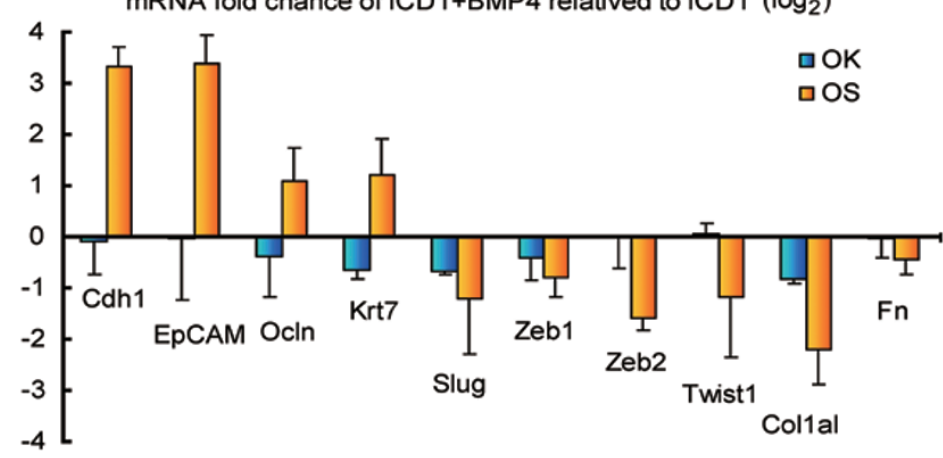

B

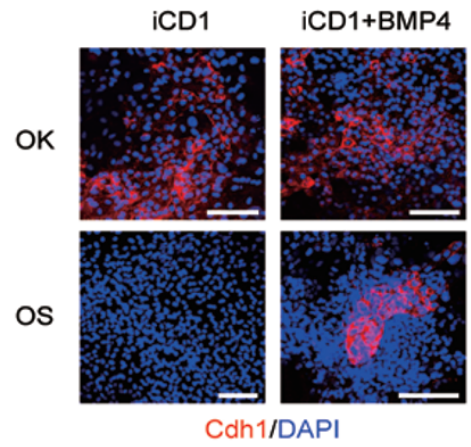

C
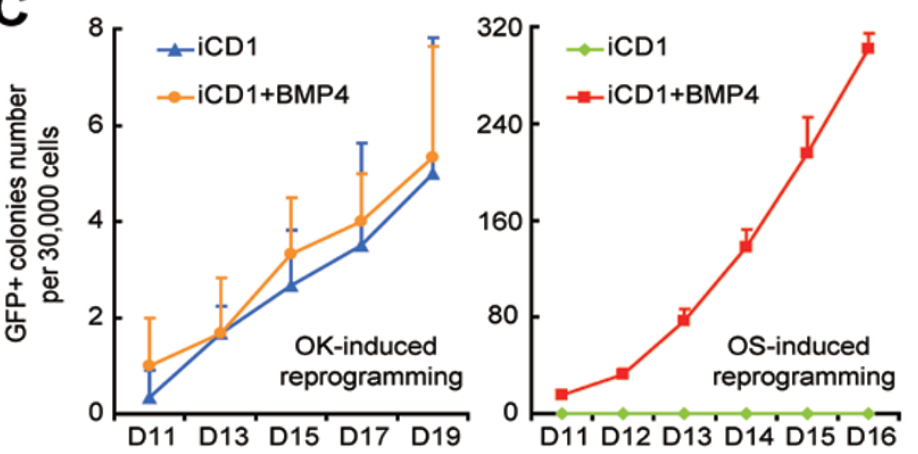

OS-mediated reprogramming at D14

iCD1

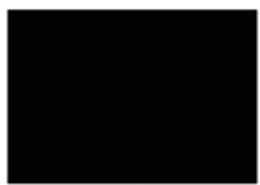

iCD1+BMP4

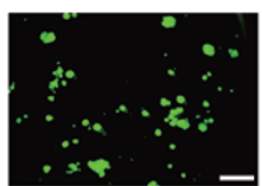

E

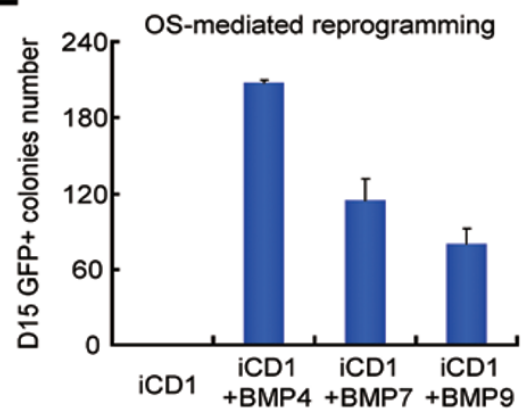

G

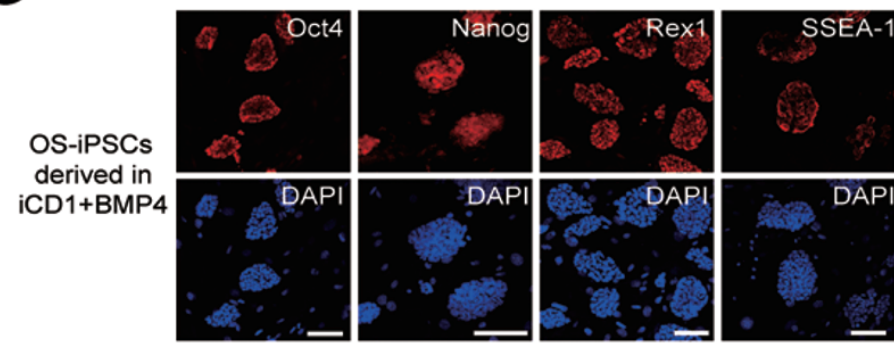

F

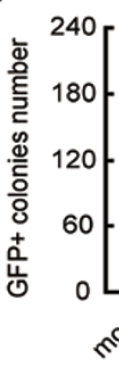

OS-mediated reprogramming

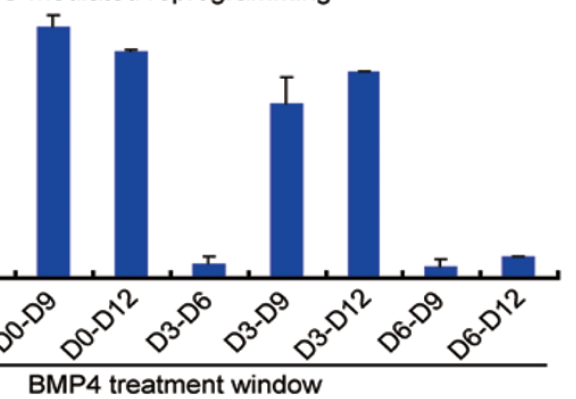

H

Figure 2 BMPs functionally replace KIf4 and support efficient reprogramming with OS. (A) Expression of indicated genes were analyzed by qRT-PCR in OK- or OS-infected MEFs at day 5 post-treatment in iCD1+BMP4 or iCD1. $n=3$. (B) BMP4 induces Cdh1 expression in OS-infected MEFs. Scale bar, $100 \mu \mathrm{m}$. (C) BMP4 enhances the reprogramming mediated by OS, but not OK. MEFs infected with OK and OS were cultured in iCD1 or iCD1+BMP4. Oct4-GFP-positive colonies were scored from day 11 post-treatment and are shown. $n=3$. (D) Representative fields of OS-infected MEFs at day 14 post-treatment in iCD1 or iCD1+BMP4 are shown. Scale bar, $2 \mathrm{~mm}$. (E) BMP7 and BMP9, similar to BMP4, enhance reprogramming mediated by OS. $n=$ 3. (F) BMP4 treatment window experiment show that D0-D9 is more sensitive to time. $n=3$. (G) OS iPSCs colonies express Oct4, Nanog, Rex1 and SSEA-1. Scale bar, $100 \mu \mathrm{m}$. (H) Germline transmission of chimeric mice generated from OS iPSCs. 
A
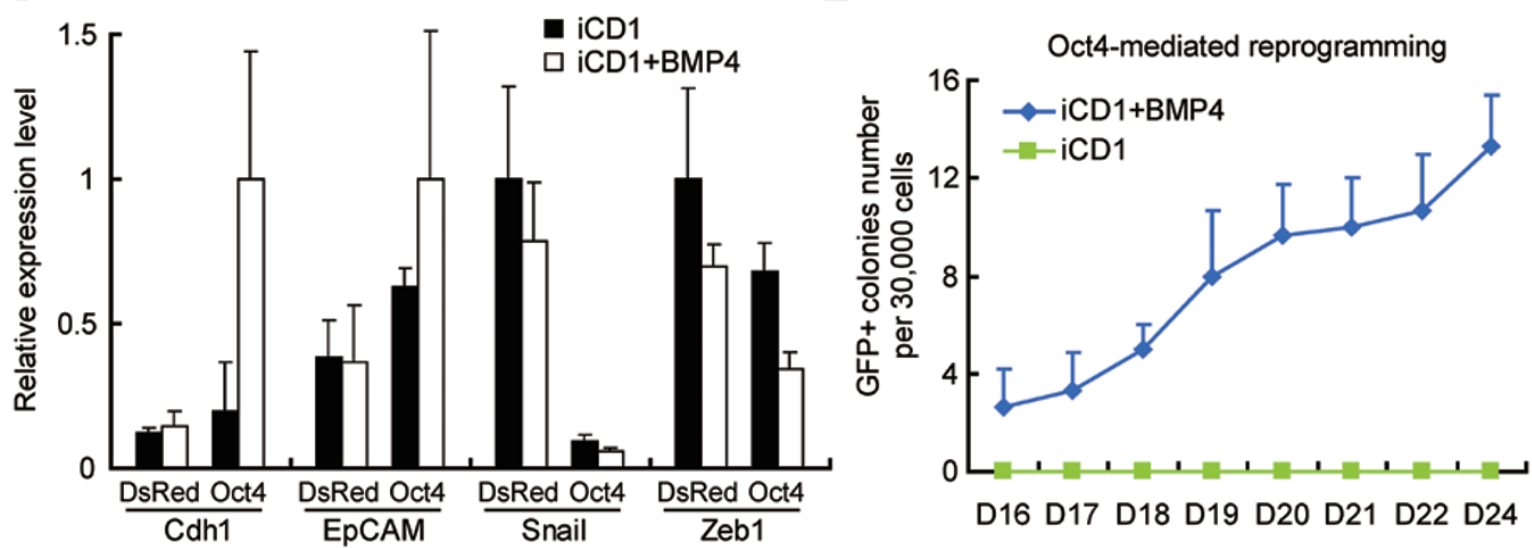

C

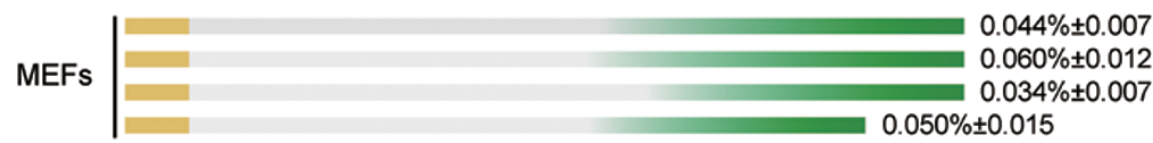

Viral transduction

Oct4-GFP colonies (-)

Oct4-GFP colonies (+)

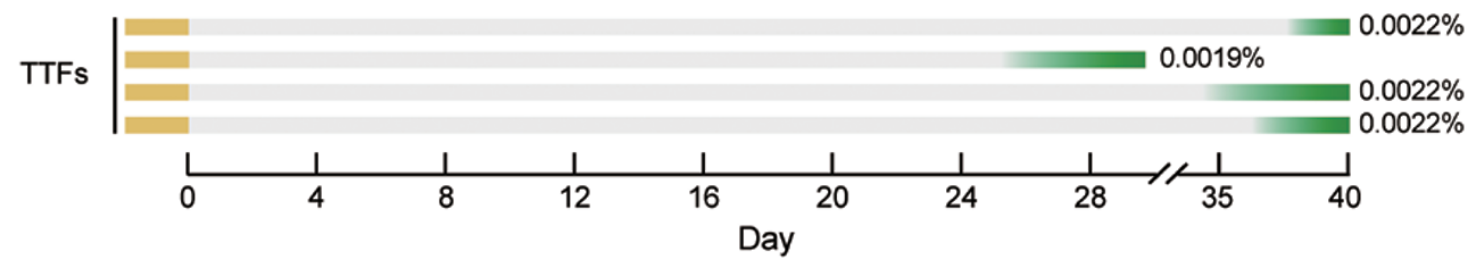

D
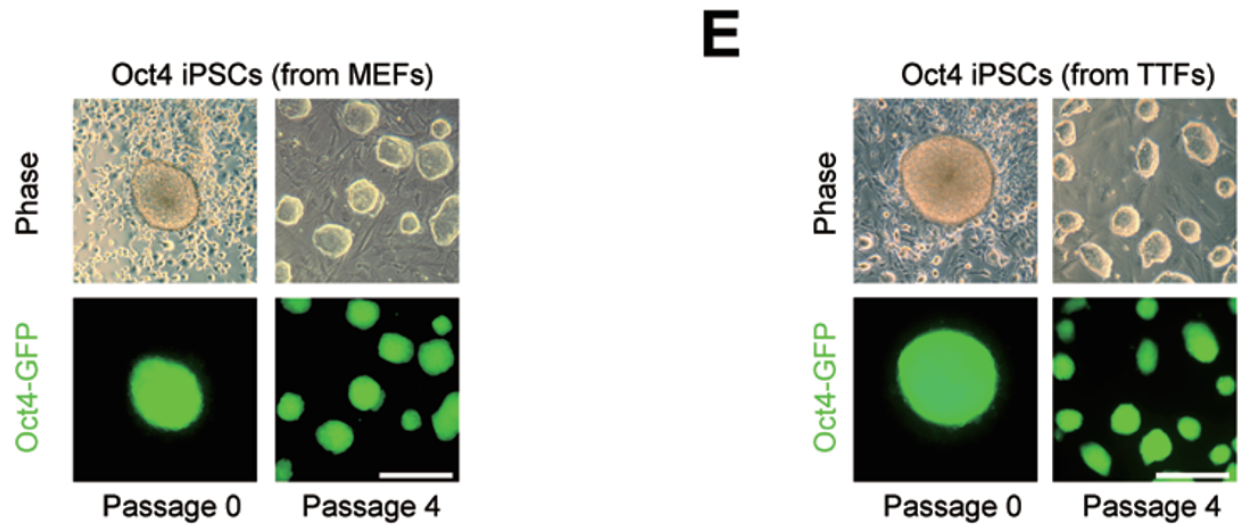

Figure 3 BMPs promote Oct4-mediated reprogramming by inducing MET. (A) BMP4 induces MET during Oct4-mediated reprogramming. MEFs infected with Oct4 or DsRed were cultured in iCD1 or iCD1+BMP4. Expression levels of Cdh1, EpCAM, Snail and Zeb1 were analyzed by qRT-PCR and the highest values of each gene were set to 1. $n=3$. (B) BMP4 enhances Oct4-mediated reprogramming. MEFs infected with Oct4 were cultured in iCD1 or iCD1+BMP4. Oct4-GFP-positive colonies were scored from day 16 to day 24 post-treatment. $n=3$. (C) BMP4 enhances Oct4-mediated reprogramming. MEFs infected with Oct4 were cultured in iCD1 or iCD1+BMP4. Oct4-GFP-positive colonies were scored from day 16 to day 24 post-treatment. $n=3$. (D) The morphology of one iPSC clone derived from MEFs by Oct4 in iCD1+BMP4. Scale bar, $250 \mu \mathrm{m}$. (E) The morphology of one iPSC clone derived from TTFs by Oct4 in iCD1+BMP4. Scale bar, $250 \mu \mathrm{m}$.

showed normal karyotypes (Figure 4C). We then characterized the integration of the Oct4 transgene by PCR and confirmed that only the Oct4 transgene was present in all Oct4-iPSC clones examined (Figure 4D). The ectopic 
A
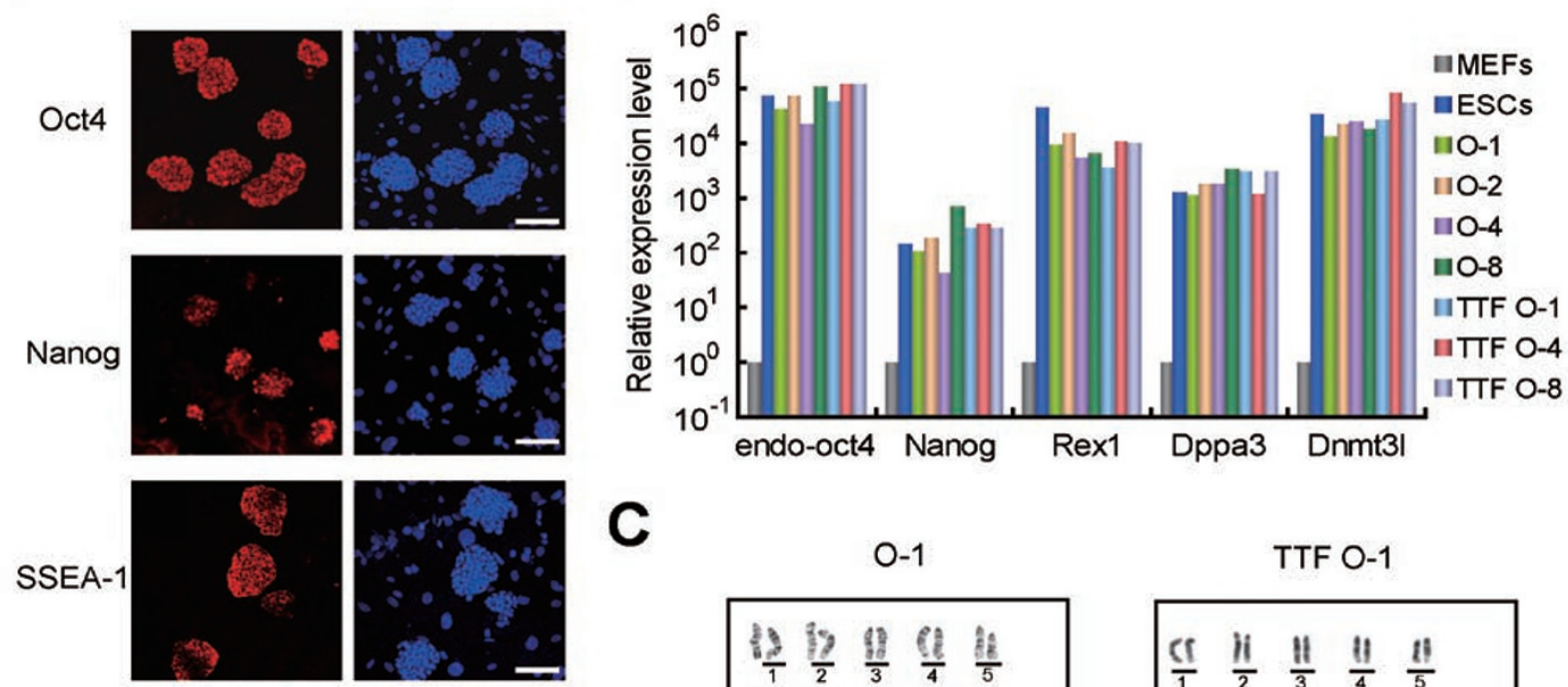

0-1

TTF 0-1
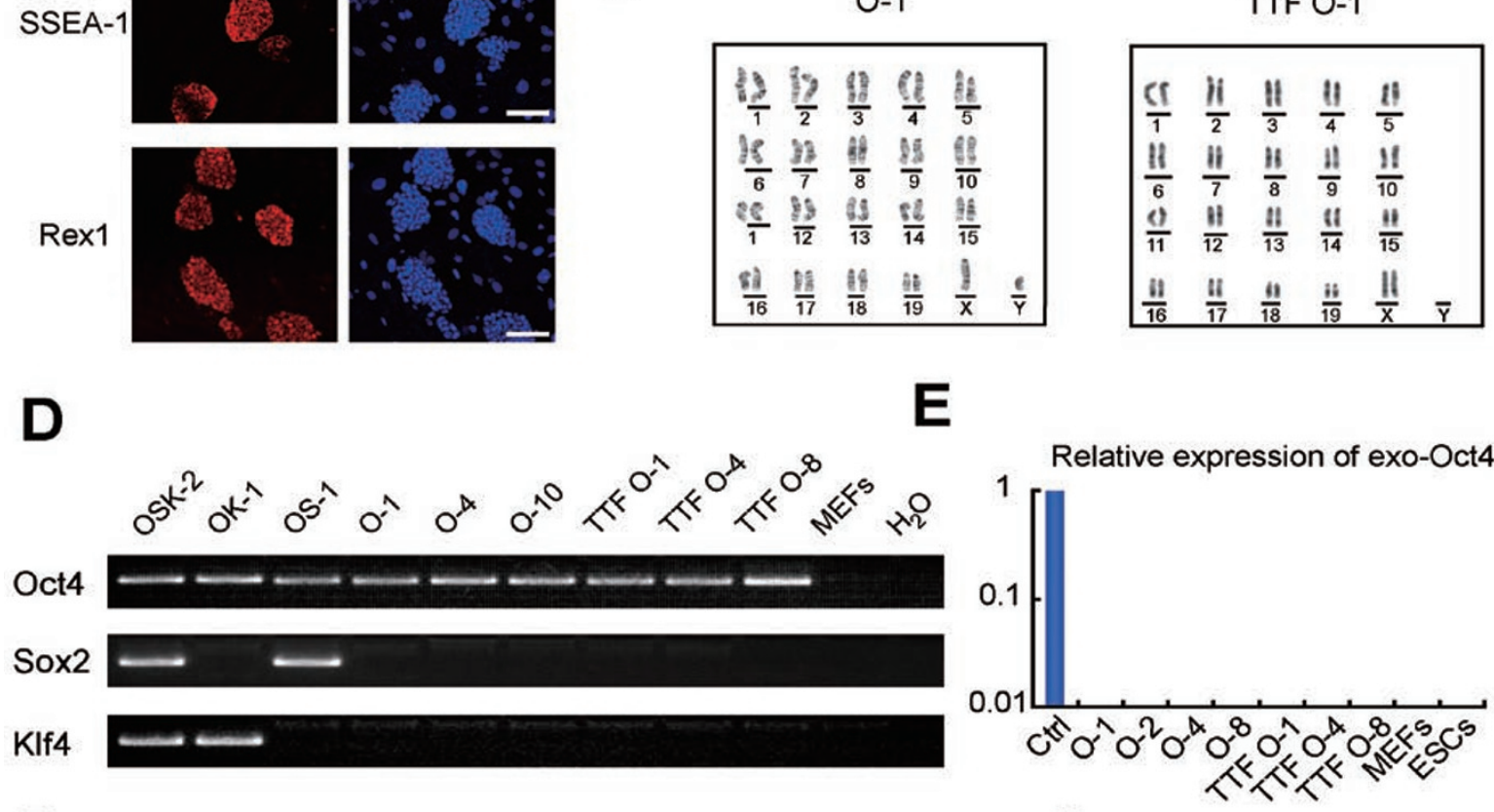

$\mathbf{F}$

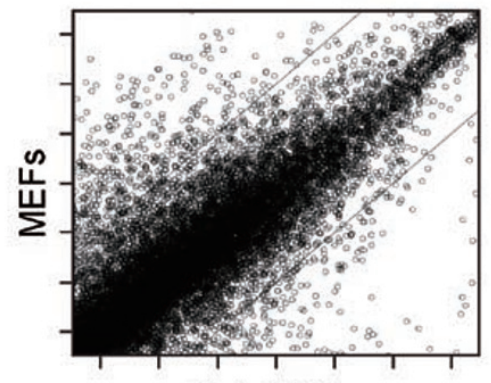

O-1 iPSCs

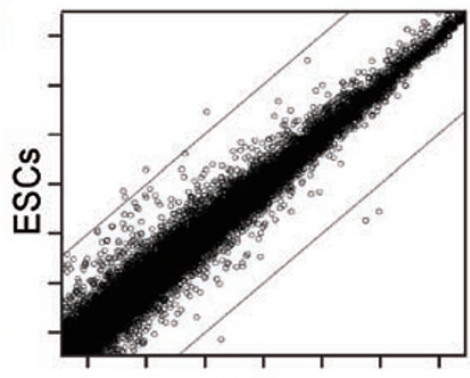

0-1 iPSCs
G

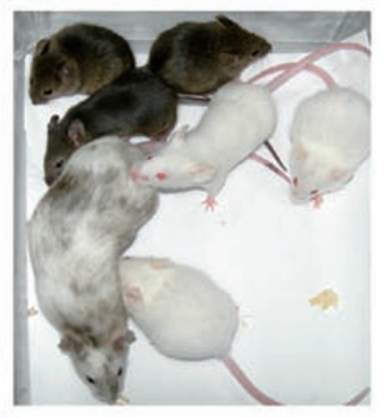

Figure 4 Oct4 iPSCs are pluripotent. (A) Oct4 iPSCs express Oct4, Nanog, SSEA-1 and Rex1 (left panels). DAPI stating was served as control (right panels). Scale bar, $100 \mu \mathrm{m}$. (B) Oct4 iPSCs express pluripotent markers. The endogenous Oct4, Nanog, Rex1, Dppa3 and Dnmt3l expression of selected iPSC clones were analyzed by qRT-PCR. Expression values were relative to that of MEFs. (C) The Oct4 iPSC clones have normal karyotype. (D) Integration analysis confirms the derivation of Oct4 iPSC clones. The presence of the retroviral transgene was examined by PCR. (E) Retroviral expression was silenced in iPSC clones. The expression of exogenous Oct4 was analyzed by qRT-PCR in selected iPSC clones. MEFs infected with OKS for 4 days were used as positive controls. (F) Global gene expression of Oct4 iPSCs is similar as that of ESCs. (G) Germline transmission of chimeric mice generated from Oct4 iPSCs. 
retroviruses were silenced in these iPSCs, indicating that they maintain pluripotency without transgene expression (Figure 4E). By global transcriptomic analysis, we showed that Oct4-iPSCs resemble ESCs and are different from MEFs (Figure 4F). The Oct4-iPSCs contributed to chimera mice when injected into blastocysts (Figure 4G and Supplementary information, Figure S3E). Furthermore, we obtained germline transmission of Oct4-iPSCs by breeding the chimeras (Figure $4 \mathrm{G}$ ). These results demonstrate that the iPSCs generated from MEFs or TTFs with Oct4 alone are fully pluripotent.

We further examined whether the replacement of Klf4 by BMP4 is mediated by the activation of endogenous Klf4 expression and showed that BMP4 does not increase the endogenous Klf4 expression in O-, OK- or OS-transduced MEFs (Supplementary information, Figure S4A). In fact, BMPs did not stimulate endogenous expression of other reprogramming factors (including OKSM and Esrrb [23]) during reprogramming (Supplementary information, Figure S4B-S4D). We then examined the expression of TGF- $\beta$ pathway members during reprogramming and showed that BMPs had inhibitory effect on Tgfbr2 and Tgfbr3, while Klf4 repressed Tgfb1, suggesting that BMPs and Klf4 inhibited the TGF- $\beta$ pathway through different mechanisms (Supplementary information, Figure S4E). So, we concluded that BMPs replace Klf4 functionally by inducing MET through a mechanism different from activating endogenous reprogramming factors.

\section{Discussion}

We showed here that Klf4 works at a rate-limiting MET step in the early phase of reprogramming by inducing epithelial markers, while Oct4 and Sox 2 contribute little to this step. Therefore, Klf4 appears to play a more important role than Sox2 during the reprogramming of mouse fibroblasts. We showed that BMPs were able

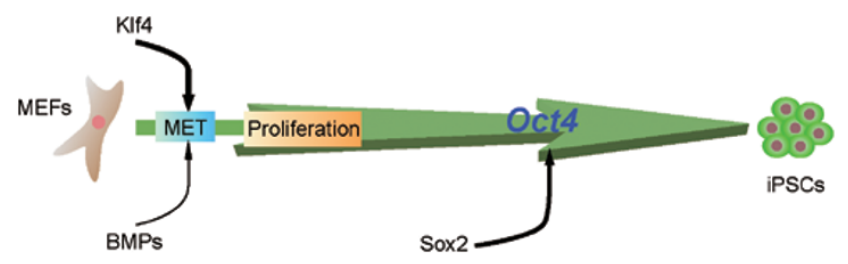

Figure 5 Relative contributions of individual reprogramming factors to reprogramming. Oct4 is the core factor sufficient for the establishment of pluripotency, and Sox2 participates to assist Oct4 in reprogramming. Klf4, which can be substituted by $\mathrm{BMPs}$, is responsible for triggering MET, an early step of reprogramming. to induce MET in Oct4- or OS-transduced fibroblasts through a different mechanism from Klf4 and can replace Klf4 functionally (Figure 5). Therefore, the effect of BMPs is context dependent on the reprogramming factors used and is beneficial only in the absence of Klf4, suggesting that BMPs are functionally redundant with Klf4 on inducing MET, or Klf4 could inhibit the function of BMPs, similar to the Wnt pathway that has a different effect on reprogramming with or without c-Myc [24]. We also showed that although Sox 2 is a core transcription factor to maintain pluripotency, it appears to only play an auxiliary role in reprogramming. Since KS could not support the generation of iPSCs (data not shown), the central role of Oct4 became apparent in the reprogramming paradigm (Figure 5). Finally, we showed that Oct4 is sufficient to reprogram MEFs and TTFs at efficiencies similar to that originally reported for Oct4, Sox2, Klf4 and Myc. Therefore, one may speculate that Oct4-mediated reprogramming can be further improved or used for screening small chemicals that can replace Oct4.

\section{Materials and Methods}

\section{Cell culture}

MEFs were cultured with DMEM+10\% FBS and ESCs/iPSCs were cultured with either mES medium (DMEM supplemented with $15 \%$ FBS, LIF and other components) or KSR medium (replace the FBS in mES medium with Knockout Serum Replacement). MEF feeder cells were inactivated by mitomycin C. BMP4, BMP7, BMP2, BMP6 and BMP9 were used at final concentrations of $10 \mathrm{ng} / \mathrm{ml}, 100 \mathrm{ng} / \mathrm{ml}, 30 \mathrm{ng} / \mathrm{ml}, 30 \mathrm{ng} / \mathrm{ml}$ and $10 \mathrm{ng} / \mathrm{ml}$, respectively. Vitamin C was purchased from Sigma (Cat No. 49752), BMPs from R\&D and CHIR99021 was synthesized at GIBH. Rest of the chemicals and reagents were purchased from Invitrogen.

\section{Generation of iPSCs}

Plasmids carrying murine Oct4, Klf4, Sox2 and c-Myc cDNA were purchased from Addgene. For direct reprogramming, Oct4GFP transgenic MEFs were plated at 4000 cells $/ \mathrm{cm}^{2}$ (for OKS combination) or $7500 \mathrm{cells} / \mathrm{cm}^{2}$ (for other combinations), and then infected with retrovirus packed by plat-E cells for two rounds. After 48 hours, the tested media were added and the day is defined as day 0 post-treatment. iPSCs were induced with iCD1, which is based on DMEM+vitamin C [25] supplemented with bFGF, CHIR99021 and other chemically defined components to support growth. The photos taken by SteREO Lumar.V12 (Zeiss) show the entire well. Reprogramming efficiency was determined by directly scoring the number of Oct4-GFP-positive colonies under microscope.

\section{Blastocyst injection}

Resulting iPSCs or ESCs were cultured in mES (containing $15 \%$ FBS) or KSR (containing 15\% serum replacement). For generation of chimaeras, iPSCs were injected into ICR blastocysts using Piezo Micro Manipulator. Injected blastocysts were transplanted into pseudopregnant ICR females. Germline transmission of resulted chimeric mice was determined by breeding F2 mouse 
with ICR mouse.

\section{Plasmid construction}

The pWP-TetOn vector was constructed by replacing the EF-1 $1 \alpha$ promoter of the pWPXLd vector with the TRE promoter of pTRETight vector (Clontech). M2rtTA from pTet-On Advanced vector (Clontech) was cloned into the pWPXLd vector.

\section{Quantitative RT-PCR}

Total mRNA was isolated using TRIzol and then was converted to cDNA. Quantitative PCR was performed and analyzed with ABI 7300. All qRT-PCR results presented in this study were from at least two independent experiments with independent viral preparations.

\section{Acknowledgments}

We are grateful to Ronghui Li, Jialiang Liang, Xiangjie Zhao, Kunlun Mo, Shilong Chu, Jieying Zhu, He Liu, Jing Li, Zhicheng Xiang, Wenzhi He, Keyu Lai, Shaoxian Sun, Haijun Xie, Lin Guo, Xiujuan Cai and Hongwen Pang for their technical assistance. We also thank all members of our laboratory for supporting our work. Data of microarray are available on GEO: GSE24930. This work is supported by the National Natural Science Foundation of China (30630039, 30725012, 90813033), MOST 973 program 2009CB941102, Ministry of Science and Technology International Technology Cooperation Program (2010DFB30430), Knowledge Innovation Project of The Chinese Academy of Sciences (KSCX2YW-R-221,KSCX2-YW-R-244) and Chinese Academy of Sciences/SAFEA International Partnership Program for Creative Research Teams.

\section{References}

1 Takahashi K, Yamanaka S. Induction of pluripotent stem cells from mouse embryonic and adult fibroblast cultures by defined factors. Cell 2006; 126:663-676.

2 Wernig M, Meissner A, Foreman R, et al. In vitro reprogramming of fibroblasts into a pluripotent ES-cell-like state. $\mathrm{Na}$ ture 2007; 448:318-324.

3 Maherali N, Sridharan R, Xie W, et al. Directly reprogrammed fibroblasts show global epigenetic remodeling and widespread tissue contribution. Cell Stem Cell 2007; 1:55-70.

4 Takahashi K, Tanabe K, Ohnuki M, et al. Induction of pluripotent stem cells from adult human fibroblasts by defined factors. Cell 2007; 131:861-872.

5 Yu J, Vodyanik MA, Smuga-Otto K, et al. Induced pluripotent stem cell lines derived from human somatic cells. Science 2007; 318:1917-1920.

6 Pei D. Regulation of pluripotency and reprogramming by transcription factors. J Biol Chem 2009; 284:3365-3369.

7 Li R, Liang J, Ni S, et al. A mesenchymal-to-epithelial transition initiates and is required for the nuclear reprogramming of mouse fibroblasts. Cell Stem Cell 2010; 7:51-63.

8 Samavarchi-Tehrani P, Golipour A, David L, et al. Functional genomics reveals a BMP-driven mesenchymal-to-epithelial transition in the initiation of somatic cell reprogramming. Cell Stem Cell 2010; 7:64-77.

9 Scholer HR, Hatzopoulos AK, Balling R, Suzuki N, Gruss P. A family of octamer-specific proteins present during mouse embryogenesis: evidence for germline-specific expression of an Oct factor. EMBO J 1989; 8:2543-2550.

10 Nichols J, Zevnik B, Anastassiadis K, et al. Formation of pluripotent stem cells in the mammalian embryo depends on the POU transcription factor Oct4. Cell 1998; 95:379-391.

11 Zeisberg M, Hanai J, Sugimoto H, et al. BMP-7 counteracts TGF-beta1-induced epithelial-to-mesenchymal transition and reverses chronic renal injury. Nat Med 2003; 9:964-968.

12 Shi Y, Desponts C, Do JT, Hahm HS, Scholer HR, Ding S. Induction of pluripotent stem cells from mouse embryonic fibroblasts by Oct 4 and Klf4 with small-molecule compounds. Cell Stem Cell 2008; 3:568-574.

13 Ichida JK, Blanchard J, Lam K, et al. A small-molecule inhibitor of TGF-Beta signaling replaces sox 2 in reprogramming by inducing nanog. Cell Stem Cell 2009; 5:491-503.

14 Kawamura T, Suzuki J, Wang YV, et al. Linking the p53 tumour suppressor pathway to somatic cell reprogramming. Nature 2009; 460:1140-1144.

15 Kim JB, Sebastiano V, Wu G, et al. Oct4-induced pluripotency in adult neural stem cells. Cell 2009; 136:411-419.

16 Maherali N, Hochedlinger K. Tgfbeta signal inhibition cooperates in the induction of iPSCs and replaces Sox2 and cMyc. Curr Biol 2009; 19:1718-1723.

17 Nakagawa M, Koyanagi M, Tanabe K, et al. Generation of induced pluripotent stem cells without Myc from mouse and human fibroblasts. Nat Biotechnol 2008; 26:101-106.

18 Judson RL, Babiarz JE, Venere M, Blelloch R. Embryonic stem cell-specific microRNAs promote induced pluripotency. Nat Biotechnol 2009; 27:459-461.

19 Chen J, Liu J, Han Q, et al. Towards an optimized culture medium for the generation of mouse induced pluripotent stem cells. J Biol Chem 2010; 285:31066-31072.

20 Wernig M, Meissner A, Cassady JP, Jaenisch R. c-Myc is dispensable for direct reprogramming of mouse fibroblasts. Cell Stem Cell 2008; 2:10-12.

21 Li W, Zhou H, Abujarour R, et al. Generation of humaninduced pluripotent stem cells in the absence of exogenous Sox2. Stem Cells 2009; 27:2992-3000.

22 Lyssiotis CA, Foreman RK, Staerk J, et al. Reprogramming of murine fibroblasts to induced pluripotent stem cells with chemical complementation of Klf4. Proc Natl Acad Sci USA 2009; 106:8912-8917.

23 Feng B, Jiang J, Kraus P, et al. Reprogramming of fibroblasts into induced pluripotent stem cells with orphan nuclear receptor Esrrb. Nat Cell Biol 2009; 11:197-203.

24 Marson A, Foreman R, Chevalier B, et al. Wnt signaling promotes reprogramming of somatic cells to pluripotency. Cell Stem Cell 2008; 3:132-135.

25 Esteban MA, Wang T, Qin B, et al. Vitamin C enhances the generation of mouse and human induced pluripotent stem cells. Cell Stem Cell 2010; 6:71-79.

(Supplementary information is linked to the online version of the paper on the Cell Research website.) 